\title{
CULTURAL MATERIAL, GLOBALIZACIÓN Y ARQUEOLOGÍA DE LA INDUSTRIALIZACIÓN
}

\author{
Juan M. Cano Sanchiz ${ }^{1}$ \\ laciudadcritica@gmail.com
}

RESUMEN: En este trabajo reflexionamos sobre la delimitación conceptual de la disciplina arqueológica desde nuestra experiencia práctica (no se trata de un texto de revisión teórica). Nuestro interés se centra en la aplicación del método arqueológico al mundo contemporáneo (en el sentido de Edad histórica); más concretamente, en la arqueología de la industrialización. Seleccionamos dos estudios de caso para ofrecer una demostración de uso transversal de herramientas de investigación y fuentes de información: el complejo minero-metalúrgico de Cerro Muriano (Córdoba, España) y los talleres de la Companhia Paulista de Estradas de Ferro en Jundiaí (São Paulo, Brasil). Estas muestras nos sirven, finalmente, para reivindicar el papel de la Arqueología y de los estudios de cultura material en el análisis de fenómenos históricos de alcance internacional, como el colonialismo económico y los procesos de globalización.

PALABRAS CLAVE: Arqueología Industrial. Interdisciplinariedad. Minería y Metalurgia. Ferrocarriles. Globalización.

RESUMO: Neste trabalho refletimos sobre a delimitação conceitual da disciplina arqueológica a partir de nossa experiência prática (este texto não é uma revisão teórica). Nosso interesse é centrado na aplicação do método arqueológico ao mundo contemporâneo (no sentido de Idade histórica); mais especificamente, este trabalho é focado na arqueologia da industrialização. Selecionamos dois estudos de caso para oferecer uma demonstração de uso transversal de ferramentas de pesquisa e fontes de informação: o complexo mineirometalúrgico de Cerro Muriano (Córdova, Espanha) e as oficinas da Companhia Paulista de Estradas de Ferro em Jundiaí (São Paulo, Brasil). Finalmente, estas amostras servem-nos para reivindicar o papel da Arqueologia e dos estudos de cultura material na análise de fenômenos históricos de abrangência internacional, tais como o colonialismo econômico e os processos de globalização.

PALAVRAS-CHAVE: Arqueologia Industrial. Interdisciplinaridade. Mineração e Metalurgia. Ferrovias. Globalização.

ABSTRACT: In this paper we aim to reflect on the conceptual framework of Archaeology from our own practical experience (this is not a theoretical review). We will focus on the application

\footnotetext{
1 Becario de Post-Doctorado FAPESP: grant \#2014/12473-3, São Paulo Research Foundation. As opiniões, hipóteses e conclusões ou recomendações expressas neste material são de responsabilidade do autor e não necessariamente refletem a visão da FAPESP.
}

( Rev. Arqueologia Pública

Campinas, SP $\quad \mathrm{v}$.

No.()

p.99-115

$/ 2015$

ISSN 2237-8294 
of archaeological methods to the study of the modern world (as an historical period); more specifically, this is a paper on archaeology of industrialization. We have selected two different case studies to offer a demonstrative example of cross-disciplinary use of research techniques and sources of information: the Cerro Muriano mining and metallurgical site (Cordoba, Spain) and the Companhia Paulista de Estradas de Ferro general workshops in Jundiaí (São Paulo, Brazil). Finally, we will use these samples to defend the role of Archaeology in the analysis of wider historical phenomena, like economic colonialism and globalization processes. KEYWORDS: Industrial Archaeology. Interdisciplinarity. Mining and Metallurgy. Railways. Globalization.

\section{REFLEXIÓNPRELIMINAR}

Este trabajo parte de concebir la Arqueología como una disciplina histórica de aplicación diacrónica cuyo campo de estudio no se delimita por coordenadas espaciales o temporales, sino metodológicas. Entendemos, como Gutiérrez Lloret (2001), que la Arqueología no se define por estudiar un determinado periodo, región geográfica o problema histórico, sino por los medios y los instrumentos empleados al hacerlo: la cultura material y la metodología arqueológica.

Esta concepción habilita las "nuevas arqueologías" surgidas a partir de la segunda mitad del siglo XX y englobadas, de manera general, en la denominada Arqueología Histórica (MAJEWSKI \& GAIMSTER, 2009); entre ellas, la Arqueología Industrial. No pretendemos ofrecer aquí una reflexión teórica sobre esta última (LABADI, 2001), ni tampoco una revisión historiográfica (CANO, 2007). Modestamente, el objetivo de este texto es fomentar el debate sobre algunas ideas que han sustentado el desarrollo práctico de nuestro trabajo.

Una de ellas ha sido entender la Arqueología Industrial como una arqueología de la industrialización o del pasado industrial, en el sentido de la "later historical Archaeology" definida por M. Palmer (2005) o de la "Archaeology of the Industrial Period" de M. Nevell (2006). Es decir, como el estudio de un periodo histórico concreto (una parte de la Historia Contemporánea), ${ }^{2}$ en lugar de un determinado tema o problema (el mundo del trabajo, con énfasis en la tecnología). De este modo, la arqueología de la industrialización, tal y como la ejercemos, aspira a ofrecer una interpretación del contexto cultural generado por la industria a partir del análisis de las huellas físicas de los procesos de industrialización (PALMER \& NEAVERSON, 1998:163), sin marginar por ello el resto de fuentes disponibles.

\footnotetext{
${ }^{2}$ Periodo que, por otro lado, no es universal. No hay una única industrialización, sino una multiplicidad de procesos que se desarrollan con diferentes intensidades y en distintos momentos en según qué partes del mundo; del mismo modo que también existen regiones no industrializadas.
}

(c) Rev. Arqueologia Pública

\begin{tabular}{l|l} 
Campinas, SP & $\mathrm{v}$.
\end{tabular}

No.()

p.99-115

$/ 2015$

ISSN 2237-8294 
Esto abre la puerta a discusiones que van más allá de la historia de la tecnología o de los conflictos sociales surgidos en los espacios de trabajo. Es el caso, por ejemplo, de los procesos de globalización inherentes a la expansión internacional de los sistemas de producción capitalista (THIESEN, 2006; HOWARD, 2011; CANO, 2012; OLIVEIRA, 2012). Esta es la idea sobre la que trabajamos en los estudios de caso que exponemos a continuación.

Finalmente, la amplitud del tema y la multiplicidad y variedad de factores (económicos, sociales, ecológicos, culturales...) que participan en los procesos de industrialización, y que cristalizan en el patrimonio industrial (SOBRINO, 2010), obligan a complementar la metodología arqueológica con otras técnicas y herramientas. O sea, es necesario plantear estudios que contemplen el uso contrastado de todas las fuentes disponibles y que aborden los problemas planteados por la historia y el patrimonio industrial desde la

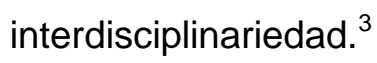

\section{ESTUDIOS DE CASO: MINERÍA Y FERROCARRILES}

\subsection{Sistema minero-metalúrgico de Cerro Muriano (Córdoba, España) ${ }^{4}$}

Cerro Muriano es un pequeña población situada unos $16 \mathrm{~km}$ al $\mathrm{N}$ de la ciudad de Córdoba

\footnotetext{
${ }^{3}$ Esta es la filosofía del Projeto Memória Ferroviária (coordinado por el Prof. Dr. Eduardo Romero de Oliveira desde la UNESP), cuyo equipo (del cual formarnos parte) trabaja de manera transversal y multidisciplinar sobre el patrimonio ferroviario e industrial del estado de São Paulo (Brasil): http://www.rosana.unesp.br/\#!/pesquisa/laboratorio-de-patrimonio-cultural/projetos/projeto-memoriaferroviaria-pmf/

${ }^{4}$ Parte de la información utilizada en este apartado procede de nuestra Tesis Doctoral (CANO, 2012). Algunos resultados ya han sido publicados (CANO, 2014; por ejemplo) y otros se encuentran en prensa (CANO, e.p.). Ahora retomamos un ejemplo concreto de transferencia de tecnología para ampliarlo con nuevos datos y ponerlo al servicio de los objetivos específicos de este trabajo. Nos interesa comparar estos resultados anteriores (minería en España) con nuestro tema de investigación actual (ferrocarriles en Brasil) para reforzar el carácter internacional y transversal de los argumentos usados en este texto.
}

( Rev. Arqueologia Pública

\begin{tabular}{l|l} 
Campinas, SP & $\mathrm{v}$.
\end{tabular}

No.()

p.99-115

$/ 2015$

ISSN 2237-8294 
(Fig. 1)

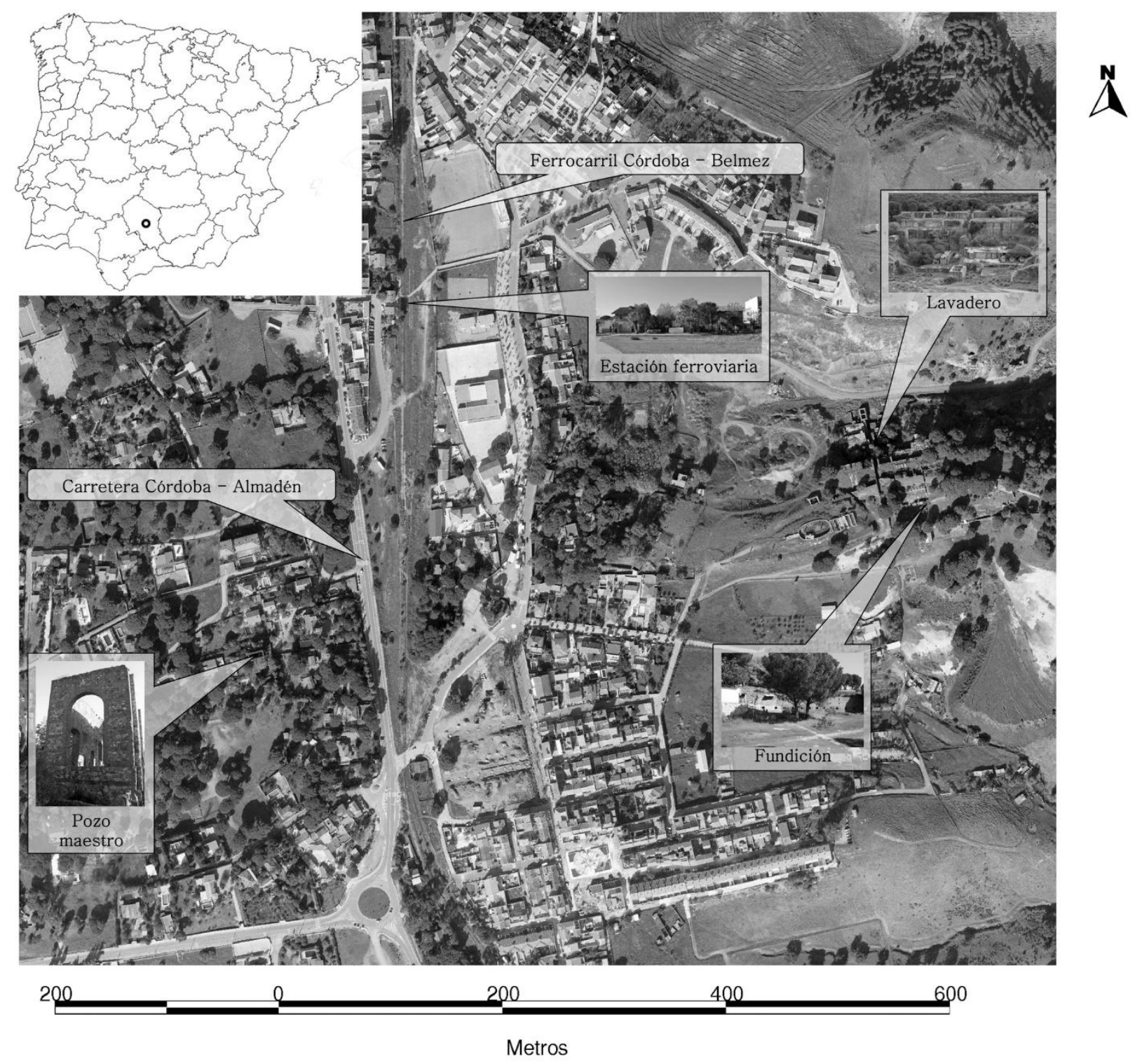

. El asentamiento, que se ubica sobre un amplio grupo de filones de cobre, acumula más de cuatro mil años de historia de explotación minera (PENCO, 2012). Aquí nos centramos en el periodo comprendido entre 1897 y 1919, cuando las minas estuvieron en manos de cuatro compañías británicas íntimamente conectadas. De entre ellas, la Cordoba Copper Co. fue la última en aparecer y también la más potente (vid. CANO, 2012 y 2014).

Los ingleses explotaron los filones de Cerro Muriano a escala industrial gracias, entre otros motivos, a una efectiva transferencia de tecnología. Sus pozos y planta de tratamiento se dotaron de equipos procedentes de la importación. Siguiendo la práctica habitual de la época,

\begin{tabular}{|l|l|l|l|l|l|l|}
\hline ( ) Rev. Arqueologia Pública & Campinas, SP & v. & No.() & p.99-115 & /2015 & ISSN 2237-8294 \\
\hline
\end{tabular}


la planta se construyó sobre una pequeña colina: la fundición en una ladera $(\mathrm{N})$ y el lavadero en otra (S). Así se aprovechaba la pendiente en los procesos de concentración del mineral (vid. CANO, 2012).

El modelo tecnológico adoptado en Cerro Muriano presentó algunas particularidades, si bien en líneas generales se adaptó a lo que podría definirse como norma internacional. Una de las más singulares fue la construcción, pionera, de un taller de concentración electromagnética que seguía la patente de la firma inglesa Murex Magnetic Co., Ltd. En Cerro Muriano se apostó por ella porque los sistemas de concentración tradicionales basados en la flotación no funcionaban debido al contenido en calcita de las menas a tratar (WALKER, 1913).

El cruce entre el registro arqueológico y las fuentes visuales disponibles permite identificar la localización exacta del taller Murex de Cerro Muriano (Fig. 2)

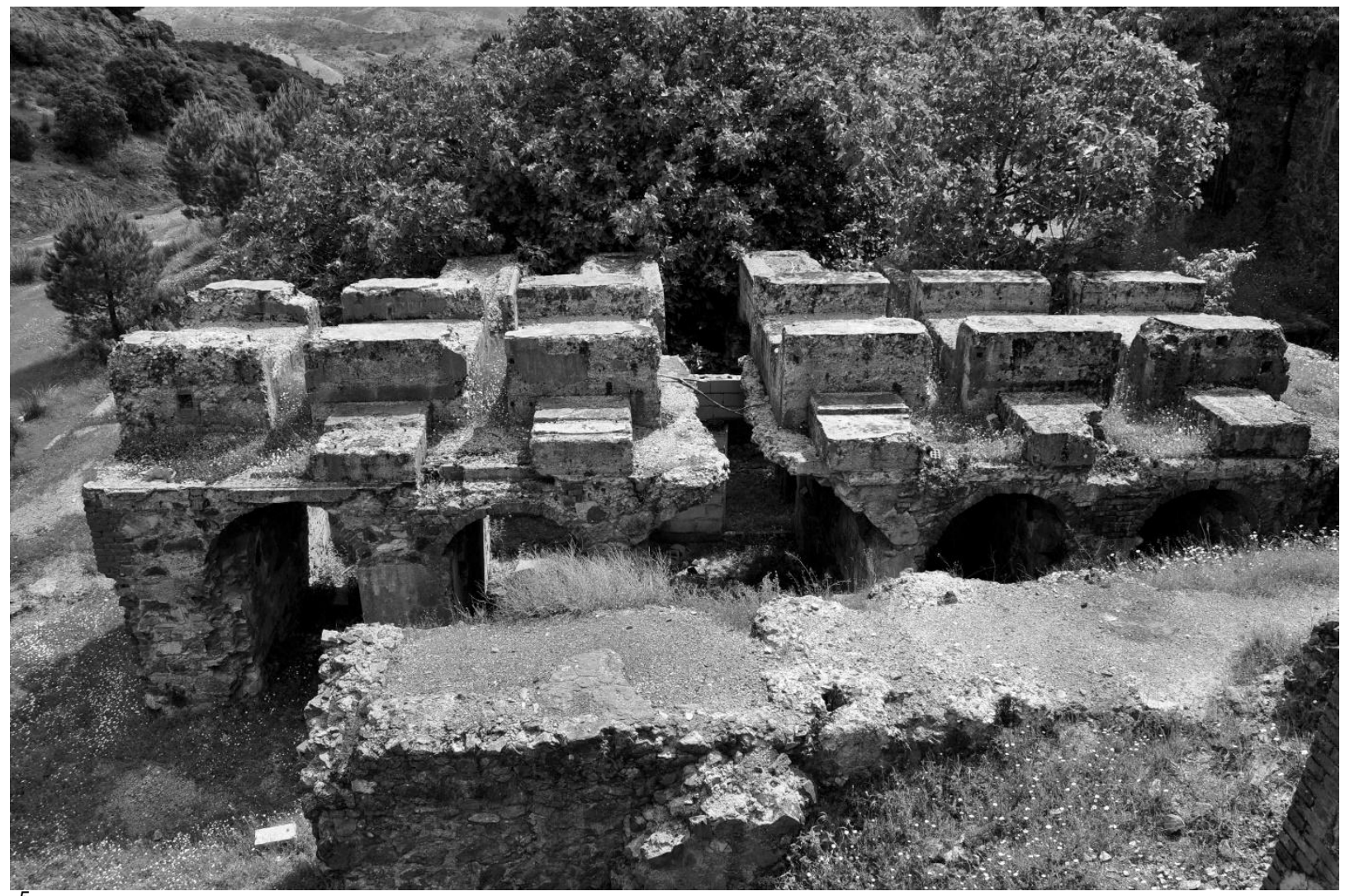

:5 se situó en la base del lavadero, al E del plano inclinado que divide la ladera y por el cual el producto concentrado era enviado a fundición. Por otro lado, los restos materiales completan los datos procedentes de las fuentes escritas. Según estas (MINING JOURNAL, 1912:257;

\footnotetext{
${ }^{5}$ Especialmente los planos diseñados por J. Cavanillas (1915).
}

C Rev. Arqueologia Pública v. p.99-115
/2015 
CAVANILLAS, 1915:43-46; MINING MAGAZINE, 1917:228), la Cordoba Copper Co. compró a lo largo del tiempo un total de nueve unidades de concentración Murex. Sin embargo, solo hay evidencia en campo para seis. Quiere esto decir que las últimas adquisiciones no estuvieron destinadas a ampliar este taller (adición de módulos nuevos), sino a renovarlo (sustitución de los antiguos).

El taller Murex también nos interesa por ser una huella física de los procesos históricos que abordamos en este trabajo. El primero es una evidente transferencia de tecnología: en Córdoba no solo fue utilizada la patente inglesa; los aparatos empleados procedían igualmente de Inglaterra (TIMES, 1909: 17), como la mayor parte del equipo de los pozos ( $v i d$. CANO, 2012).

El hecho de que la producción de esta planta fuera enviada a Inglaterra (CARBONELL \& LÓPEZ, 1946:212) nos permite plantear un segundo fenómeno: ${ }^{6}$ el colonialismo económico. En Cerro Muriano encontramos un grupo de capitalistas ingleses que emplea tecnología importada para explotar los recursos naturales de un territorio extranjero con el único objetivo de sacarlos del país. Las firmas inglesas allí desplazas no tuvieron interés en el desarrollo del entorno, del mismo modo que tampoco se aprecia un arrastre significativo de la minería en la economía cordobesa (vid. CANO, 2014).

Finalmente, transferencia de tecnología y colonización económica se sitúan, en este caso, en la base de otro proceso global: la homogeneización internacional de los sistemas de extracción, producción y distribución industrial (CANO, 2012). En Cerro Muriano esto no solo es rastreable en la circulación de maquinarias; también en los flujos de ideas y de personas (vid. CANO, 2014:138 ss). Sus minas fueron una de las primeras en el mundo en poner en práctica el sistema de concentración Murex Magnetic Co. Contemporáneamente, este era adoptado en Broken Hill (Nueva Gales del Sur, Australia). Ambos territorios tuvieron asimismo en común una relación directa con el reputado ingeniero holandés G. D. Delprart (MAWSON, 1958), así como otra más tangencial con el británico Goodchild (TIMES, 1909:17; MINING JOURNAL, 1910:693). Aun cuando podrían señalarse otros muchos puntos de conexión (incluyendo individuos, países y empresas diferentes), creemos que lo expuesto basta para plantear que la movilidad de las personas fue un factor determinante en el mencionado proceso de homogeneización.

\footnotetext{
${ }^{6}$ El contexto internacional generado por la Primera Guerra Mundial obligó a los ingleses a comercializar su cobre en España (MINING JOURNAL, 1917:164), pero este nunca fue su plan inicial.
}

(C) Rev. Arqueologia Pública

\begin{tabular}{l|l} 
Campinas, SP & $\mathrm{v}$.
\end{tabular}

No.()

p.99-115

$/ 2015$ ISSN 2237-8294 
Por otro lado, llama la atención que una empresa dedicada a la concentración de minerales escogiera para auto-representarse el nombre de murex, un tipo de molusco gasterópodo marino. Un análisis transversal de historia económica e imagen publicitaria puede ofrecer una explicación al respecto. Entre los hombres que financiaron la creación de la Murex Magnetic Co. hubo algunos miembros de la familia británica Samuel (THE KALGOORLIE MINER, 1909:3; MINING \& SCIENTIFIC MAGAZINE, 1910:646), más conocida por haber fundado The "Shell" Transport and Trading Co. en 1897; el nombre de la firma procedía del antiguo negocio familiar: el comercio de conchas marinas para coleccionistas (HOWARD, 2011:124). Tal vez el murex fuese escogido para establecer un vínculo icónico entre una iniciativa empresarial nueva (Murex Magnetic) y otra de reputación internacional (Shell) (Fig. 3)

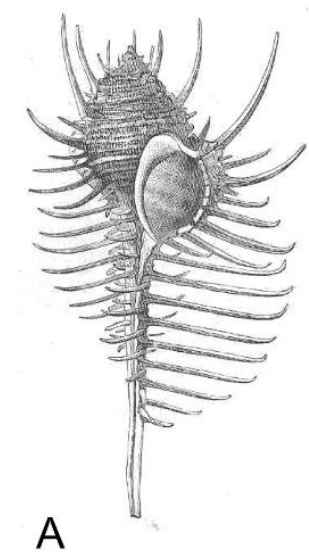

A

B

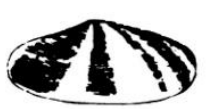

1900

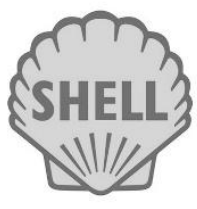

1955

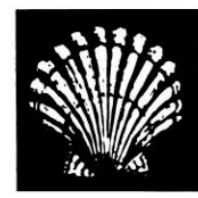

1904

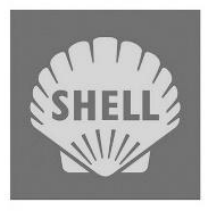

1961

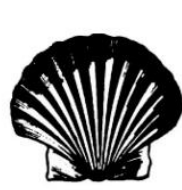

1909

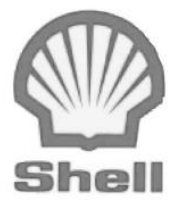

1971

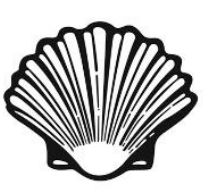

1930

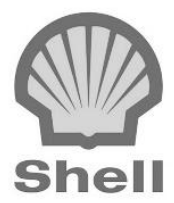

1995

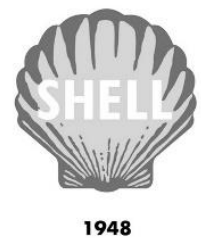

1948

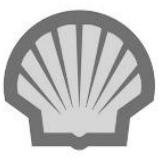

1999

- Tampoco debe olvidarse que Murex fue, precisamente, el nombre con el que los Samuel bautizaron al primer buque petrolero que atravesó el Canal de Suez, botado en 1892 (BAMBERG, 2000:290). En cualquier caso, cuando laMurex Magnetic Co. fue fundada en 1909 (THE TIMES, 1909:17), The "Shell" Transport \& Trading Co. ya se había fusionado con la Royal Dutch Petroleum Co.,en un intentó de hacer competencia a la potente Standard Oil norteamericana. Tras estas grandes firmas se situaban, respectivamente, dos familias de riqueza y poder: los Rothschild y los Rockefeller (HOWARD, 2011:124-125). De nuevo, personas en red como base para un proceso de globalización.

\subsection{Complejo de la Companhia Paulista de Estradas de Ferro en Jundiaí (São Paulo, Brasil)}

Jundiaí es una ciudad de la Región Metropolitana de São Paulo, localizada unos 50 km al NO de la capital. Se trata de un enclave relevante en la historia ferroviaria del estado, pues

( Rev. Arqueologia Pública

\begin{tabular}{l|l} 
Campinas, SP & v.
\end{tabular}

No.()

p.99-115

$/ 2015$

ISSN 2237-8294 
constituyó el término de la primera línea paulista: la Santos-Jundiaí, abierta al tráfico por la São Paulo Railway en 1867 (PINTO, 1903:35). En 1872 la Companhia Paulista de Estradas de Ferro unió esta ciudad con Campinas (PINTO, 1903:40), iniciándose así, con la aparición de nuevas vías y empresas, la incursión del tren hacia el interior del estado (MATOS, 1990: 59 ss).

La Companhia Paulista también erigió en Jundiaí sus principales talleres de montaje y mantenimiento de locomotoras y vagones, junto a los que situó sus oficinas y otros edificios auxiliares y administrativos (Fig. 4)

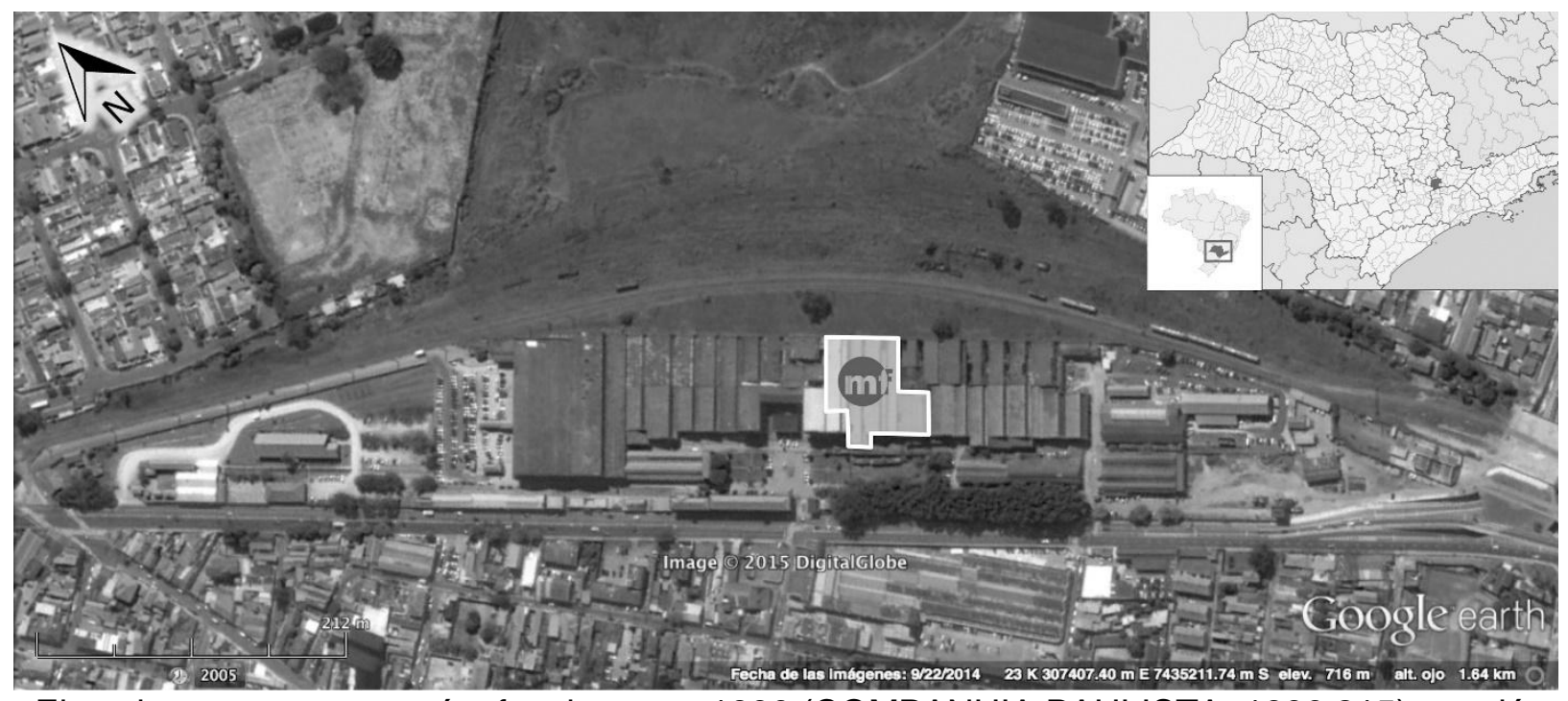

. El conjunto, que comenzó a funcionar en 1896 (COMPANHIA PAULISTA, 1896:215), creció y evolucionó a lo largo del tiempo en función de las innovaciones técnicas del sector (OLIVEIRA, 2012). En 1971 pasó a manos de la empresa pública Ferrovia Paulista S.A., que lo mantuvo en uso hasta 1998 (CAZZOLATTO \& ACUIO, 2013). Hoy es conocido como Complejo FEPASA y ha sido parcialmente reocupado para alojar nuevas actividades ( $v i d$. BATISTA, 2013).

En la actualidad desarrollamos un estudio de caso sobre un sector concreto de este conjunto que corresponde, grosso modo, a los talleres erigidos inicialmente para la reparación de locomotoras a vapor (vid. Fig. 4). ${ }^{7}$ Aquí nos centramos en sus cubiertas.

\footnotetext{
${ }^{7}$ A ferrovia como agente de globalização: estudo arqueológico transversal do Complexo das Oficinas da Companhia Paulista em Jundiaí (FAPESP 2014/12473-3; Supervisor: Prof. Dr. E. Romero de Oliveira). Nuestro trabajo se vincula al Projeto Memória Ferroviária, igualmente coordinado por el profesor Romero.
}

(C) Rev. Arqueologia Pública

Campinas, SP $\quad$ v.

No.()

p.99-115

$/ 2015$ ISSN 2237-8294 
El espacio abordado se compone de cinco naves paralelas de distintas dimensiones. A E y O están cerradas por los muros de ladrillo (sobre zócalo de piedra) de las fachadas laterales del complejo. El interior es diáfano. Cada nave cuenta con su propia cubrición, que es del tipo diente de sierra, con dos aguas (la N mayor que la S) y una luz entre estas (que también discurre en diagonal). La función portante recae sobre un grupo de pilares de acero roblonado, así como en los muros de cierre. Sobre los primeros apoyan una serie de cuadros de acero, que sostienen a su vez la armadura de madera que hace de cama a los materiales del tejado (Fig. 5)
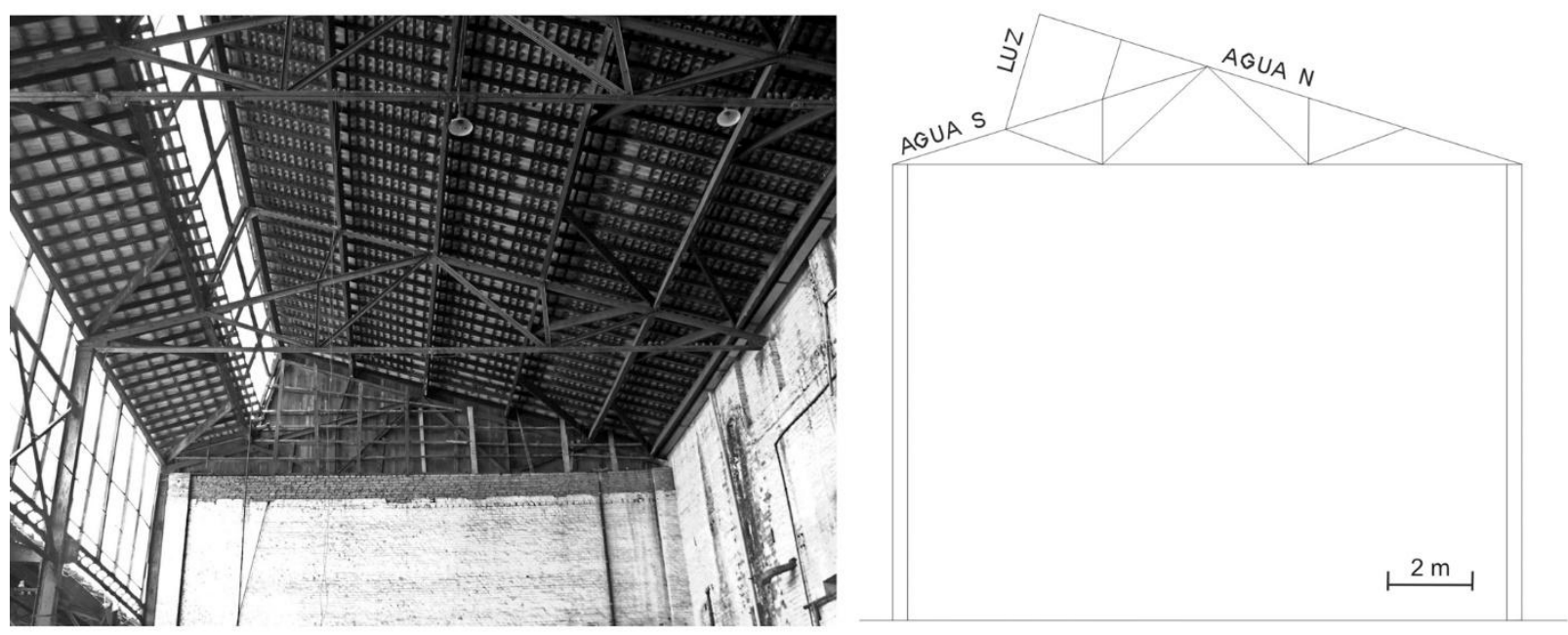

Las cubiertas se cierran con tejas cerámicas planas (aguas) y vidrio (luz). En las naves más septentrional y meridional ${ }^{8}$ de la muestra, el plástico ha sustituido al vidrio y el fibrocemento a la teja cerámica. ${ }^{9}$ Estas alteraciones, que no son las únicas apreciables en dichas naves, evidencian una evolución del espacio de trabajo que puede relacionarse con ciertos cambios funcionales y tecnológicos (que no podemos detenernos a explicar aquí). ${ }^{10}$

Por otro lado, las cubiertas de estos talleres también nos sirven para reflexionar sobre los procesos de transferencia de tecnología y globalización que nos interesan en este

${ }^{8}$ De la que solo consideramos su sector occidental, pues el oriental queda fuera de nuestra área de estudio.

${ }^{9}$ En el agua $\mathrm{S}$ de la nave $\mathrm{N}$ sí se emplean tejas cerámicas, pero no son las originales.

${ }^{10}$ Ya hemos planteado algunas hipótesis de interpretación al respecto en sendos textos enviados para su publicación al n. 25 de Anales de Arqueología Cordobesa (en evaluación) y a los Anais da VII Semana Nacional de Museus na UNIFAL-MG (en prensa).

(c) Rev. Arqueologia Pública

Campinas, SP

v. No.() p.99-115 $/ 2015$ ISSN 2237-8294 
trabajo. ${ }^{11}$ Según la documentación gráfica disponible en la Biblioteca del Museo de la Compañía Paulista, ${ }^{12}$ toda la estructura portante fue diseñada y fabricada en el exterior. En los planos de obra de las cubiertas originales, firmados en 1892, puede leerse el nombre de dos compañías diferentes: The Phoenix Bridge Co. y Fry, Miers \& Co. La primera era una empresa de Phoenixville (Pensilvania, EE.UU.) especializada en la construcción de puentes y viaductos de hierro (y de acero), así como de otras estructuras metálicas (HAER, 1994:11-12). La segunda, con base en Londres, era una firma de ingeniería que actuaba en Brasil como agencia mediadora en el encargo y la supervisión de la edificación de diversas infraestructuras ferroviarias. ${ }^{13}$ De este modo, la Compañía Paulista confió a empresas del exterior tanto el diseño y la fabricación de la estructura metálica de las naves como la gestión y el control de su construcción.

Las tejas constituyen otro elemento interesante en esta línea de discusión. Dentro de la muestra de estudio existen varios derrumbes que se sitúan exactamente en los puntos donde las cubiertas han cedido. Dichos derrumbes están compuestos casi en su totalidad por fragmentos de tejas cerámicas planas de la casa Arnaud Etienne et Cíe. (Saint-Henri, Marsella, Francia), ${ }^{14}$ que también se observan in situ en varios puntos de los tejados. Hay registro en las memorias anuales (relatórios) de la Companhia Paulista (1893:169) de la compra en 1892 de 91.332 "telhas francezas" para los talleres de Jundiaí, entonces en construcción. ${ }^{15}$ Debe tratarse, por tanto, de las originales.

Junto a ellas se detectan, de manera más dispersa y en un número mucho menor, restos de tejas de fabricación brasileña. A partir de sus fragmentos pueden identificarse con seguridad varias compañías cerámicas del estado de São Paulo

\footnotetext{
${ }^{11}$ En este caso, nuestras hipótesissiguen la fundamentación teórica del Projeto Memória Ferroviária y se complementan con los resultados ya generados por nuestros colegas de equipo (OLIVEIRA, 2012; DELVIZIO \& OLIVEIRA,2013; etc.). También son deudoras de las ideas de otros compañeros que trabajan desde otras áreas de conocimiento, como Mónica Ferrari (arquitectura industrial), Domingo Cuéllar (historia económica) o Julián Sobrino (patrimonio industrial); y especialmente de las de nuestro supervisor, Eduardo Romero de Oliveira. A todos ellos mostramos nuestra gratitud.

${ }^{12}$ Biblioteca do Museu da Companhia Paulista, Reserva, Caja 358 (varios planos). Agradecemos la colaboración de A. Torrejais y del personal de la biblioteca.

${ }^{13}$ Grace's Guide. British Industrial History. Disponible en: http://www.gracesguide.co.uk/Fry, Miers and Co (acceso: 30/04/2015).

${ }_{11}^{14}$ Tal y como puede leerse en las propias tejas.

${ }^{15} \mathrm{La}$ fuente no recoge el nombre del fabricante. Es importante advertir que este tipo de teja plana era generalmente conocido como teja francesa, por ser el país galo su principal productor. Con todo, pensamos que las de Jundiaí fueron efectivamente importadas de Marsella, pues el nombre de dicha ciudad aparece en los fragmentos analizados.
}

(c) Rev. Arqueologia Pública

\begin{tabular}{l|l} 
Campinas, SP & v.
\end{tabular} No.() p.99-115 12015 ISSN 2237-8294 
느 II SEMANA DE

$\bigcirc$ ARQUEOLOGIA

त DA UNICAMP

HISTÓRIA E CULTURA MATERIAL: DESAFIOS DA CONTEMPORANEIDADE

ATAS
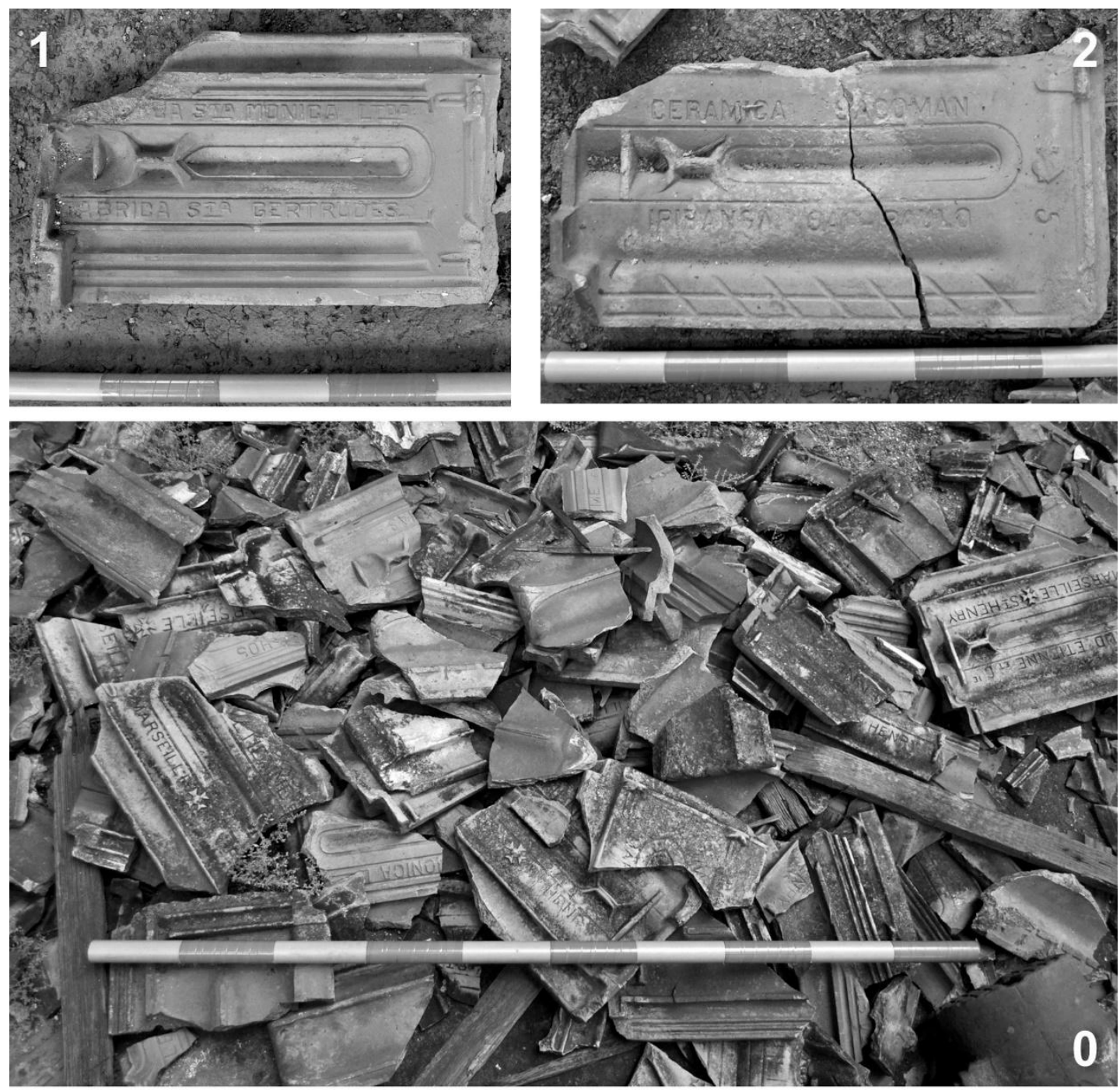

(Fig. 6)
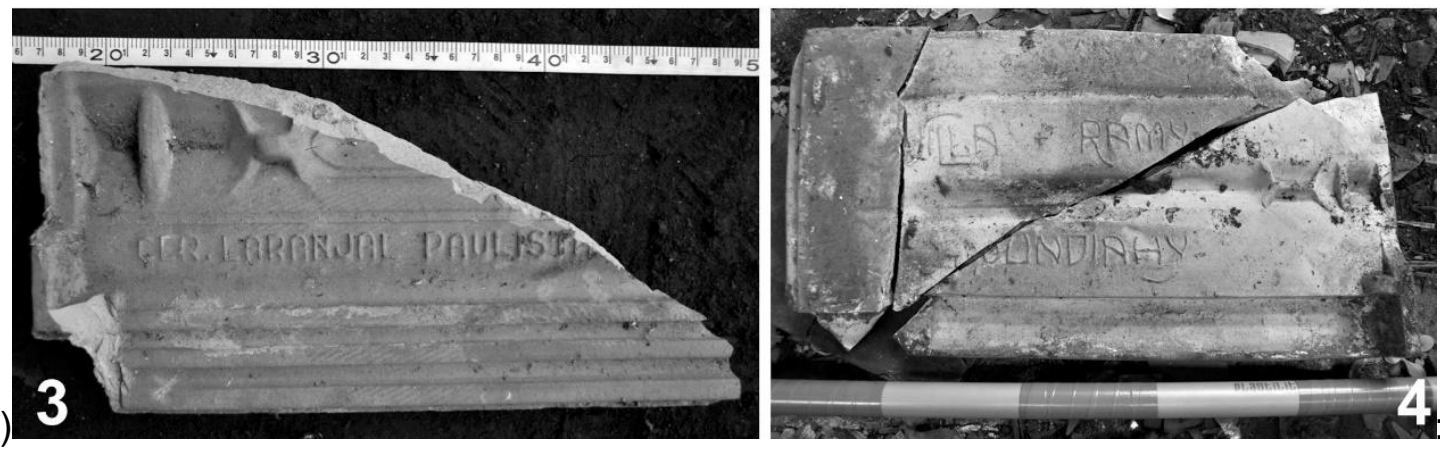

O Grupo Cerâmico Laranjal Paulista (Laranjal Paulista), Cerâmica Santa Mónica Ltda. (Santa

$\overbrace{\substack{0 \\ 0 \\ 0 \\ 0 \\ 0}}^{0}$

Gertrudes), Cerâmica S. José (Campinas), Villa Ramy (Jundiaí), Cerâmica Sacoman (Ipiranga, São Paulo) y Olaria S. Anna (Vallinhos). No hay referencias en los relatórios de la Compañía Paulista a ninguna de ellas.

(c) Rev. Arqueologia Pública

\begin{tabular}{|l|l} 
Campinas, SP & $\mathrm{v}$.
\end{tabular}

\begin{tabular}{l|l} 
v. & No.()
\end{tabular}

\begin{tabular}{|l|l} 
p.99-115 & $/ 2015$
\end{tabular}

ISSN 2237-8294 
En nuestra opinión, estas tejas nacionales pudieron adquirirse de manera puntual a lo largo del tiempo para realizar reparaciones. La hipótesis es interesante, pues podría indicar un cambio de tendencia en la Compañía Paulista. Esta comenzó a funcionar con una fuerte dependencia de la tecnología extranjera (OLIVEIRA, 2012), pero más tarde pudo desarrollar cierta relación (aunque fuera débil) con el territorio y con otras industrias brasileñas emergentes, especialmente a medida que mejoraba y se ampliaba la red de transporte estatal (MATOS, 1990:159 ss). Sea como fuere, la presencia de firmas foráneas y materiales arquitectónicos importados en Jundiaí hacen de los talleres de la Companhia Paulista un testigo físico de la homogeneización internacional de los espacios de producción (y del consecuente proceso de globalización vinculado a la industrialización) que pretendemos poner en evidenciar con estos estudios de caso.

\section{REFLEXIÓN FINAL: PEQUEÑOS OBJETOS QUE CUENTAN GRANDES HISTORIAS}

La historia industrial ha sido interpretada tradicionalmente desde la lectura exclusiva de las fuentes escritas, a pesar de las limitaciones de estas (imposibilidad de registrarlo todo) y de la subjetividad inherente a la fabricación de un texto (que es siempre una acción humana). La cultura material tiene capacidad para ofrecer un punto de vista diferente, aportar datos nuevos y contrastar o complementar la información que recogen el resto de fuentes (CERDÀ, 2008). En el caso de Cerro Muriano, el cruce de los datos procedentes de los documentos textuales y del registro arqueológico determina, como ha sido expuesto, que el taller Murex experimentó varias reformas a lo largo del tiempo. En Jundiaí, el método arqueológico permite identificar al fabricante de las "telhas francezas" empleadas en la cubierta original y también detectar la incorporación, en un segundo momento, de materiales de producción nacional.

Como hemos tratado de demostrar, la Arqueología puede hacer que pequeños objetos (un bloque de hormigón en Cerro Muriano o un fragmento de teja en Jundiaí) cuenten grandes historias. Para ello, no obstante, precisa de la interacción metodológica con otras disciplinas y del uso contrastado de todas las fuentes disponibles. Solo así se consigue vislumbrar, a partir de una huella física, la existencia de un determinado proceso de globalización basado en la homogeneización internacional del espacio industrial durante los siglos XIX y XX; en el sentido de que es posible rastrear los mismos objetos, técnicas de trabajo y hasta personas en diversas partes del planeta (HOWARD, 2011; ROMERO, 2012; CANO, 2012). El problema, claro está, es más complejo (BEAUDRY, 2007:303 ss), pero confiamos en que esta sencilla 
aportación sirva para reivindicar la voz de la Arqueología en el debate sobre el papel jugado por los procesos de industrialización en la construcción del mundo contemporáneo.

\section{REFERENCIAS}

- BAMBERG, J. British Petroleum and Global Oil 1950-1975: the challenge of Nationalism. Cambridge: Cambridge University Press, 2000, 637 p.

- BATISTA, S. S. S. O Complexo Cultural FEPASA em Jundiaí: histórico e perspectivas. In: ACUIO, L. G. L.; CAZZOLATTO, T. H. (Org.), Revitalização do Patrimônio Ferroviário através de Eventos Culturais. Jundiaí: Edições Brasil, 2013. p. 45-86.

- BEAUDRY, M. C. Concluding Comments. Revolutionizing Industrial Archaeology? In: CASELLA, E.; SYMONDS, J. (Eds.), Industrial Archaeology. Future Directions. Nueva York: Springer, p. 301-312, 2007.

- CANO SANCHIZ, J. M. Arqueólogos en la Fábrica. Breve recorrido por la historiografía de la Arqueología Industrial. Spal. Revista de Prehistoria y Arqueología, Sevilla: n. 16, p. 5367, 2007 . La minería y la metalurgia del cobre como elementos de industrialización: análisis arqueológico del complejo británico de Cerro Muriano (Córdoba). Córdoba: Servicio de Publicaciones de la Universidad de Córdoba, 450 p., 2012. Tesis Doctoral. Programa de Doctorado en Arqueología, Universidades de Córdoba, Huelva, Málaga y Pablo de Olavide (Sevilla), Córdoba, 2012.

- Cobre, colonialismo económico y globalización: la inversión británica en las minas de Cerro Muriano (Córdoba, 1897-1919). Revista de Historia Industrial, Barcelona: n. 56, p. 115-149, 2014. . La explotación inglesa de las minas de Cerro Muriano (Córdoba, España). Una historia de colonialismo económico de principios del siglo XX. Oxford: Archaeopress (British Archaeological Reports, International Series), en prensa, $246 \mathrm{p}$.

- CARBONELL TRILLO-FIGUEROA, A.; LÓPEZ DE AZCONA, J. M.Criaderos de cobre de la provincia de Córdoba. Madrid: Instituto Geológico y Minero de España, documento mecanografiado, 1946, p. 311.

- CAVANILLAS, J. Memoria sobre las minas de Cerro Muriano. Madrid: Escuela Especial de Minas de Madrid (E.T.S.I. de Minas U.P.M.), carpeta con varios documentos, 1915. 
- CAZZOLATTO, T.H.; ACUIO, L. G. L. Históricos da Companhia Paulista de Estradas de Ferro e do Complexo FEPASA em Jundiaí: do auge à decadência. In:ACUIO, L.G. L.; CAZZOLATTO, T. H. (Org.), Revitalização do Patrimônio Ferroviário através de Eventos Culturais. Jundiaí: Edições Brasil, 2013, p. 33-41.

- CERDÀ, M.Arqueología Industrial. Teoría y práctica. Valencia: Universitat de Valencia, 2008, $260 \mathrm{p}$.

- COMPANHIA PAULISTA. Relatório n. 38 da Companhia Paulista para a sessão de Assembléa Geral em 30 de septembro de 1888. São Paulo: Typographia a vapor de Jorge Seckler \& Comp., 1888, 147 p. . Relatório n. 44 da Directoria da Companhia Paulista de Vias Ferreas e Fluviaes para a sessão de Assembléa Geral de 1893. São Paulo: Typ. da Companhia Industrial de São Paulo, 1893, 268 p.

.Relatório n. 47 da Directoria da Companhia Paulista de Vias Ferreas e Fluviaes para a sessão de Assembléa Geral em 30 de abril de 1896. São Paulo: Typ. da Companhia Industrial de São Paulo, 1896, 339 p.

- _ Relatório n. 71 da Directoria da Companhia Paulista de Estradas de Ferro para a sessão de Assembléa Geral em 26 de junho de 1920. São Paulo: Casa Vanorden, 1920, $125 \mathrm{p}$.

- DELVIZIO, I.A.; OLIVEIRA, E. Corpus de relatórios da Companhia Paulista de Vias Ferreas e Fluviaes (1885 a 1897): levantamento da terminologia ferroviária com o programa WordSmith Tools". In:I Colóquio Nacional de Língua, Documentos e História, Fortaleza: EDUECE, p. 329-342, 2013.

- GUTIÉRREZ LLORET, S. Arqueología: introducción a la historia material de las sociedades del pasado. $2^{\underline{a}}$ ed. (1 ${ }^{\underline{a}}$ ed. 1997). Alicante: Universidad de Alicante, 2001, 224 p.

- HAER (Historic American Engineering Record). Phoenix Bridge (Bridge No. 6386). Photographs, written historical and descriptive data. HAER $n$. VA-105. Philadelphia: HAER, 1994, 20 p. Disponible en: http://lcweb2.loc.gov/master/pnp/habshaer/va/va1700/va1740/data/va1740data.pdf (acceso en 30/04/2015).

- HOWARD, M. C. Transnationalism and Society: an introduction. Londres: McFarland \& Co., 2011, 321 p. 
- $\quad$ LABADI, S. Industrial Archaeology as Historical Archaeology and Cultural Anthropology. Papers from the Institute of Archaeology, Londres: n. 12, p. 77-85, 2001.

- MAJEWSKI, T.; GAIMSTER, D. (Eds). International Handbook of Historical Archaeology. Nueva York: Springer, 2009, 698 p.

- MATOS, O. N.Café e ferrovia: a evolução ferroviária de São Paulo e o desenvolvimento da cultura cafeeira.4 ${ }^{\mathrm{a}}$ ed. (1 ${ }^{\mathrm{a}}$ ed. 1974). Campinas: Pontes,1990, 178 p.

- MAWSON, P. A vision of steel: the life of G. D. Delprat, C.B.E., General Manager of B.H.P. 1898-1921. Melbourne: F. W. Chesire, 1958, 269 p.

- $\quad$ MINING JOURNAL. Murex Magnetic. The Mining Journal, Londres: 28/05/1910, p. 693, 1910.

- _ _ _ C C _ _ _ Cordoba Copper. The Mining Journal, Londres: 12/03/1912, p. 257, 1912.

- _ C Cordoba Copper Company, Ltd. The Mining Journal, Londres: 24/03/1917, p. 164-165, 1917.

- $\quad$ MINING MAGAZINE. Cordoba Copper. The Mining Magazine, Londres: v. XVI, n. 5, p. 227228, 1917.

- $\quad$ MINING \& SCIENTIFIC MAGAZINE. Interesting Magnetic-Concentration Process. Mining \&Scientific Magazine, San Francisco: v. 101, n. 20, p. 646, 1910.

- NEVELL, M. The 2005 Rolt Memorial Lecture. Industrial Archaeology or the Archaeology of the Industrial Period? Models, Methodology and the Future of Industrial Archaeology. Industrial Archaeology Review, Leeds: v. XXVIII, n. 1, p. 3-15, 2006.

- OLIVEIRA, E. R. Electrificação em empresas ferroviárias paulistas: aspectos da tecnologia e da industrialização em São Paulo (1902-1937). In: CAPEL, H.; CASALS, V.; CUÉLLAR, D. (Eds.),La electricidad en las redes ferroviarias y la vida urbana: Europa y América (siglos XIX y XX). Madrid: Fundación de los Ferrocarriles Españoles, p. 187-201, 2012.

- PALMER, M. Understanding the Workplace: A Research Framework for Industrial Archaeology in Britain. Industrial Archaeology Review, Leeds: v. XXVII, n. 1, p. 9-17, 2005.

- PALMER, M; NEAVERSON, P. Industrial Archaeology. Principles and Practice. Londres: Routledge, 1998, $180 \mathrm{p}$.

- PENCO VALENZUELA, F. Historia de la minería en Córdoba: Cerro Muriano, Sitio Histórico. Córdoba: Almuzara, 2010, 284 p.

- PINTO, A. A.Historia da Viação Publica de S. Paulo.São Paulo: Typ e Papelaria de Vanorden \& Cia., 1903, 322 p. 
- SOBRINO SIMAL, J. Los archivos del trabajo en la estrategia de un plan para la recuperación del patrimonio industrial de Andalucía.Arch-e. Revista Andaluza de Archivos, Sevilla: n.2, p. 121-132, 2010.

- THE KALGOORLIE MINER. The Murex Magnetic process. The Kalgoorlie Miner, Kalgoorlie: 1/07/1909, p. 3, 1909.

- THE TIMES. Murex Magnetic Company (Ltd). The Times, Londres: 20/06/1909, p. 17, 1909.

- THIESEN, B. V. Arqueologia industrial ou arqueologia da industrialização? Mais que uma questão de abrangência. Patrimônio. Revista Eltrônica do Iphan, n. 4, s/p, 2006. Disponible en: http://portal.iphan.gov.br/portal/baixaFcdAnexo.do?id=520 (acceso en 04/05/2015).

- WALKER, E. Flotation Process During 1913. Mining and Scientific Press,San Francisco: v. 108, n. 1(3/01/1914), p. 79-80, 1914.

v. No.() 


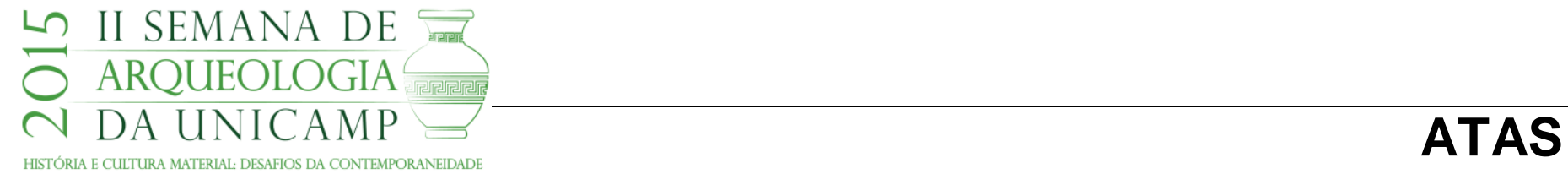

(2)

\begin{tabular}{|l|l|l|l|l|l|l|}
\hline (c) Rev. Arqueologia Pública & Campinas, SP & v. & No.() & p.99-115 & /2015 & ISSN 2237-8294
\end{tabular} 